Chen J., Dai F., and Chen Z. (2017). “Assessing Construction Workers' Vigilance Level Through Wearable Electroencephalography System", In: LC3 2017: Volume I - Proceedings of the Joint Conference on Computing in Construction (JC3), July 4-7, 2017, Heraklion, Greece, pp. 45-52. DOI: https://doi.org/10.24928/JC3-2017/0030.

\title{
ASSESSING CONSTRUCTION WORKERS' VIGILANCE LEVEL THROUGH WEARABLE ELECTROENCEPHALOGRAPHY SYSTEM
}

\author{
Jiayu Chen ${ }^{1}$, Fei Dai ${ }^{2}$, and Zejun Chen $^{3}$
}

\begin{abstract}
Construction industry requires constant caution on construction labors when they expose to hazardous environments. Although received fundamental safety training, construction workers tend to insensitive to hazards because of their long time exposures to risks. Many construction workers take unsafe behavior when they wrongly estimated the potential risks. Therefore, the discrepancy between the environment risks and workers' perceived risks is the major cause of unsafe behaviors. However, current assessing approaches are subjective and post-hoc. In this paper, we proposed a wearable Electroencephalography (EEG) system to quantitatively and objectively assess the construction workers' vigilance level for perceived risks. With such data acquisition approach, the construction workers' risk perception can be further understood and guide the safety training programs in future.
\end{abstract}

Keywords: Construction safety, EEG, vigilance, wearable.

\section{INTRODUCTION}

Safety is one of the most significant concerns of contractors in construction projects. Many studies suggest construction labours' unsafe behaviours is the most frequently observed reason of accidents (Bohm \& Harris 2015; Fang et al. 2016). In Suraji et al.'s study on the construction accident reports, $88 \%$ of the accidents is the results of workers' inappropriate operations (Suraji et al. 2001). Haslam et al. categorize the unsafe behaviors into three types: (1) overlooking safety due to heavy workloads and other priorities; (2) taking shortcuts to save effort and time; (3) inaccurately perceiving risk, with feelings of invulnerability and "it won't happen to me." (Haslam et al. 2005). Recent neuroscience study suggests human's risk-Sensitivity subject to the reinforcement-learning of human brain (Niv et al. 2012). Therefore, long time exposure to the hazardous environment without accident makes construction workers insensitive to potential risks over time. The negligence of safety hazards is mainly due to the incorrect estimation and lack of vigilance towards existing risks. Many training programs are designed to raise the attention of workers on various types of hazards, however, it is extremely difficult to track the learning outcomes in practice because of the lack of proper assessment approaches.

Currently, the majority of vigilance level assessments utilize questionnaires to collect worker's subjective feelings (Suraji et al. 2001), but some researchers suspect the objectivity and reliability of the questionnaire methods (DeJoy 2005). Therefore, there is a research gap to develop a more objective and quantitative measurement framework on

Assistant Professor, Department of Architecture and Civil Engineering, City University of Hong Kong, Hong Kong, jiaychen@cityu.edu.hk

2 Assistant Professor, Department of Civil and Environmental Engineering, West Virginia University, Morgantown, WV, USA, fei.dai@mail.wvu.edu

3 Research Assistant, School of Business, Tung Wah College, 21/F, 98 Shantung Street, Kowloon, Hong Kong,13005403@twc.edu.hk 
workers' perceived risks. In this paper, we proposed a wearable Electroencephalography (EEG) system to monitor construction worker's brain signals. With the model of vigilance level assessment, we hope to fill the aforementioned research gap with a more rigorous and reliable monitoring system to assess construction workers' risk perception. With the proposed approach, project managers will able to design proper training programs and identify risk-insensitive individual on construction site.

\section{BACKGROUND}

\subsection{Risk Perception and Construction Safety}

Understanding how workers perceive and assess risks is extremely important for construction safety management, since the perceived risk determines how they respond to the risks (Arezes \& Miguel 2008; Krallis \& Csontos 2006). Deery studied drivers' risk perception when they confront various hazards and summarized this process into four steps: hazard detection, risk perception/acceptance, self-assessment, and action (Deery 1999). People tend to be opportunism when they assume the risk is minor in the hazardous environment and themselves are skilful enough to avoid risk (Bohm \& Harris 2015; Stewart et al. 2012). In Bohm and Harris' study, the measured "objective risk" shows a huge difference with the workers' self-reported risks (Bohm \& Harris 2015). Many factors affect the risk perception ability of a person, such as experience, concentration, and emotion (Krallis \& Csontos 2006). Dzeng et al. studied the construction workers' ability in hazard inspection and found that comparing to novice workers, the experience workers are more consistent and faster in risk assessment (Dzeng et al. 2016). Working memory/ concentration level is another factor affect the workers' detection of risk stimuli in the surrounding environment (Chen et al. 2016). When workers devote more working memory and vigilant to the tasks associated risks, they can avoid injuries and end up with nearmiss accidents (Yang et al. 2016). Therefore, many risk-taking or unsafe behaviors is the results of mismatching between subjective perceived risks and objective actual risks.

Based upon these findings, many researchers suggest hazard recognition training to help workers develop proper understanding on hazards and correct their unsafe behaviors (Stewart et al. 2012; Rethi et al. 1999). After training, researchers developed questionnaires to understand the risk perception capacity of construction workers and future improve these programs (Cheng et al. 2012). However, accurately assessing the workers' perceived risk or vigilance level at various hazardous conditions is extremely difficult.

\subsection{Risk Perception and EEG}

Working memory (mental workload) is the major cognitive resource for risk perception for construction workers (Sweller 1988). When people are more vigilant, there is more working memory allocated to precaution; when they are less vigilant, more working memory is allocated to work activities (Chen et al. 2016; Mitropoulos \& Namboodiri 2010). Based-on such rationale, researchers proposed both subjective and objective measurement approaches. NASA Task Load Assessment (NASA-TLX) is one of the most widely used subjective attention measurement framework developed by the US National Aeronautics and Space Administration (Hart \& Staveland 1988). Recent objective measurement approaches utilize neuroimaging techniques with functional magnetic resonance imaging (fMRI) and EEG (Olbrich et al. 2009). Although fMRI equipment is more reliable and accuracy, due to its bulky size and formidable price, wearable EEG shows better engineering potential. 
Previous studies suggest EEG brain signals have a significant correlation with human mental activities (Pfurtscheller \& Silva 1999; Sterman \& Mann 1994). In addition, the raw EEG signals can be decomposed to signals at various frequency bands, which associated with different brain activities (Demos 2005; Buzsaki 2011). These rhythms can be categorized into five frequency bands based on a dichotomous division.

- Delta waves $(1-3 \mathrm{~Hz})$ : associated with unconsciousness, deep sleep, or catalepsy;

- Theta waves $(4-7 \mathrm{~Hz})$ : associated with creativity, spontaneity, distractibility, inattention, daydreaming, depression, and anxiety;

- Alpha waves $(8-12 \mathrm{~Hz})$ : associated with physical and mental relaxation as well as awareness of one's surroundings;

- Beta waves (13 - $30 \mathrm{~Hz})$ : associated with focusing, analysis, conscious alertness, tension, and fear;

- Gamma waves $(31-50 \mathrm{~Hz})$ : associated with problem solving, learning, and facing cognitive challenges.

Energy or power spectral densities (PSDs) is a valid indicator for the energy distribution among each frequency bands. Different PSDs reflect which part of the brain is activated and a subject's concentration level (Cohen 2014). Lin et al. also suggested that the spectral dynamics of EEG was strongly related to declining vigilance (Lin et al. 2012). Olbrich et al. proposed a vigilance stage model and utilized PSDs of different EEG frequency bands to classify the vigilance level (Olbrich et al. 2009). In this research, we propose to employ wearable EEG to automatically monitor the vigilance states of construction workers. These vigilance states are the direct and quantitative representation of the perceived risks of workers.

\section{METHODOLOGY}

\subsection{On-site Experiment}

\subsubsection{Experiment Design}

To examine the proposed method, we designed an experiment to gather the construction workers' EEG response to different hazardous conditions. We invited real construction workers who have different year of experiences and type of trades to test the consistency of their perceived risks.

The task in the experiment is moving two mental tubes to a predefined material deposit. On the path to the destination, we set up several obstacles. There were four obstacles in total including (Obs 1) a pile of wood boards, (Obs 2) an overhead rebar, (Obs 3) few iron tubes, and (Obs 4) a pile of rebar. The wood boards pile was around $30 \mathrm{~cm}$ high; the overhead rebar had a height of $160 \mathrm{~cm}$; the iron tubes and rebar pile were close to each other, so the subjects needed to pass both of them consequently in a short time. All the subjects were required to relax for a few seconds first and then pick up the iron tubes. They are required to wear an EEG monitoring headset and repeat the task for three times.

\subsubsection{Subjects and Apparatus}

Ten healthy construction workers participated in this study, and all of them were male (mean age $51.8 \pm 3.7$ years), right-handed, and fully rested before the experiment. Two of the subjects have more than 20 year experience, seven of them have working experience less than 7 years, and one recently joint the crew. Three of them were mason and rest of 
them were choreman. All participants were well informed of the nature of this study, which was approved by the university human ethics review committee.

In the experiment, we utilized Emotive EPOC+ as the EEG monitoring device [56]. The EPOC+ is multi-channel EEG monitoring headset with 14 fixed channels and $128 \mathrm{~Hz}$ sampling rate. The applying location of all channels followed the internal 10-20 system, including AF3, F7, F3, FC5, T7, P7, O1, O2, P8, T8, FC6, F4, F8, and AF4. The entire the experimental procedures were recorded with a camera for further video analysis.

\subsection{Vigilance Assessment with EEG signal processing}

The most well established vigilance assessment framework is EEG-vigilance stages model, which divides the vigilance level based on the dominant alpha bands and those with lowvoltage non-alpha bands (Loomis et al. 1937; Roth 1961; Hegerl et al. 2008). The summary of vigilance stages and their frequency features is listed in the Table 1.

Following vigilance stage shows the human perceived risk based on their EEG PSD distribution. In this research, we focus on the spatial performance of different brain regions at different vigilance level and outcome consistency among different workers.

Table 1: Vigilance stages

\begin{tabular}{|c|c|c|c|}
\hline $\begin{array}{l}\text { Vigilance } \\
\text { Stage }\end{array}$ & Behaviors & Alpha Band Power & EEG Patterns \\
\hline A1 & $\begin{array}{c}\text { relaxed } \\
\text { wakefulness }\end{array}$ & \multirow{5}{*}{$\begin{array}{c}\text { alpha power }(8-12 \mathrm{~Hz}) \text { of }(\mathrm{F} 3 \\
\mathrm{F} 4, \mathrm{O} 1, \mathrm{O} 2)^{*}>50 \% \text { of total power } \\
(2-12 \mathrm{~Hz}) \text { of delta, theta and } \\
\text { alpha }\end{array}$} & \multirow{2}{*}{$\begin{array}{l}\text { alpha power }(\mathrm{O} 1+\mathrm{O} 2)>55 \% \text { of } \\
\text { alpha power }(\mathrm{F} 3+\mathrm{F} 4+\mathrm{O} 1+\mathrm{O} 2)\end{array}$} \\
\hline $\mathrm{A} 2$ & $\begin{array}{c}\text { relaxed } \\
\text { wakefulness }\end{array}$ & & \\
\hline A3 & $\begin{array}{c}\text { relaxed } \\
\text { wakefulness }\end{array}$ & & $\begin{array}{l}\text { alpha power }(\mathrm{F} 3+\mathrm{F} 4)>55 \% \text { of } \\
\text { alpha power }(\mathrm{F} 3+\mathrm{F} 4+\mathrm{O} 1+\mathrm{O} 2)\end{array}$ \\
\hline B1 & drowsiness & & $\begin{array}{l}\text { total power }(\mathrm{F} 3+\mathrm{F} 4+\mathrm{O} 1+\mathrm{O} 2)-\text { alpha } \\
\text { power }(\mathrm{F} 3+\mathrm{F} 4+\mathrm{O} 1+\mathrm{O} 2)<200 \mu V^{2}\end{array}$ \\
\hline $\begin{array}{l}\text { B2 } \\
\text { B3 }\end{array}$ & $\begin{array}{l}\text { loss of } \\
\text { conscious }\end{array}$ & & $\begin{array}{l}\text { Total Power }(\mathrm{F} 3+\mathrm{F} 4+\mathrm{O} 1+\mathrm{O} 2)-\text { alpha } \\
\text { power }(\mathrm{F} 3+\mathrm{F} 4+\mathrm{O} 1+\mathrm{O} 2)>=200 \mu V^{2}\end{array}$ \\
\hline
\end{tabular}

${ }^{*} \mathrm{~F} 3, \mathrm{~F} 4, \mathrm{O} 1$ and $\mathrm{O} 2$ are index of EEG channels

\section{RESULTS AND DISCUSSION}

\subsection{Experiment Results and Raw Data}

The experiment results were grouped as different actions including (1) Relax; (2) Pick up; (3) Avoid Obs 1; (4) Avoid Obs 2; (5) Avoid Obs 3 and 4. The raw data sets were tagged based on the action events. All subjects were indexed for each trial. Since the timefrequency analysis normally focuses on one individual for the spatial analysis, subject 10 was selected since he had the least working experience. The statistical analysis utilizes data of all trials (10 subjects and each subject had three trials).

\subsection{Identification of Vigilance Stages and Spatial Analysis}

The raw data was processed with Fourier Transformation to calculate the PSD of F3, F4, $\mathrm{O} 1$ and $\mathrm{O} 2$ channels. Based on the EEG-vigilance stages model, Table 2 reports the 
identified vigilance levels for the subject 10 . Then this identified results were compared with spatial analysis to evaluate the response at different functional regions of the brain.

Table 2: Identified Vigilance Stage of the Subject 10

\begin{tabular}{ccccccc}
\hline $\begin{array}{c}\text { Subject } \\
\text { Index }\end{array}$ & $\begin{array}{c}\text { Trial } \\
\text { Index }\end{array}$ & Action 1 & Action 2 & Action 3 & Action 4 & Action 5 \\
\hline \multirow{2}{*}{10} & 1 & A3 & A2 & A2 & A1 & A1 \\
& 2 & A3 & A1 & A2 & A3 & A1 \\
& 3 & A3 & A3 & A2 & A1 & A1 \\
\hline
\end{tabular}

Following Figure 1 shows the spatial PSD of the subject. Comparing across actions, it can be observed that comparing to relaxation activity, the obstacle avoiding activates shows significant differences in various channels. Different from pick up activity, the obstacle avoiding activities stimulate the frontal and middle region activated. Right polar seems more sensitive to risk identification comparing to left polar.

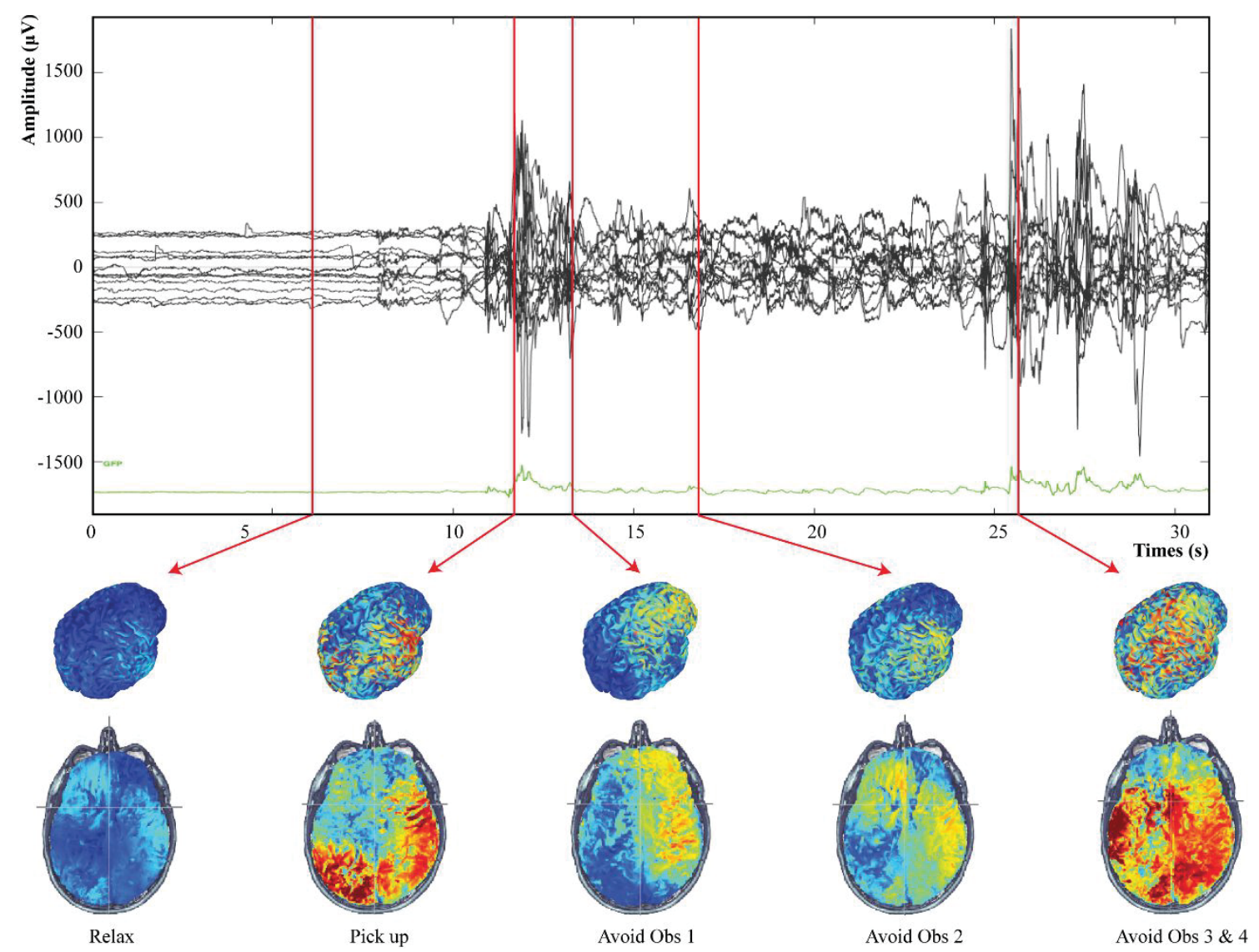

Figure 1: Spatial PSD of Different Actions for Subject 10

\subsection{Statistical Analysis}

To avoid the bias caused by individual differences, we also conducted a statistical analysis for all trials. Since the identified EEG-vigilance stages are categorical, a multinomial logistic regression was performed. Table 3 shows part of the analysis results. 
Table 3: Multinomial Logistic Regression on Actions and EEG channels (Partial)

\begin{tabular}{ccccc}
\hline & \multicolumn{3}{c}{ Model 1 } & \multicolumn{2}{c}{ Model 2 } \\
\cline { 2 - 5 } & StageA1/A3 & StageA2/A3 & StageA1/A3 & StageA2/A3 \\
\hline Intercept & 311.00 & 199.28 & 49.03 & -27.18 \\
Pick up & $14.24^{* *}$ & $10.74^{* *}$ & & \\
Avoid Obs1 & $12.96^{* *}$ & $13.54^{* *}$ & & \\
Avoid Obs2 & $18.09^{* *}$ & $17.66^{* *}$ & & \\
Avoid Obs3\&4 & $10.04^{*}$ & $10.10^{* *}$ & & \\
\hline O1 & -8.99 & 51.24 & -16.31 & 59.10 \\
O2 & -389.10 & -256.74 & -55.24 & 12.52 \\
F3 & $290.15^{* *}$ & $202.78^{* *}$ & $42.24^{*}$ & $82.89^{* *}$ \\
F4 & 220.66 & 233.41 & 30.50 & \multicolumn{2}{c}{76.29} \\
\hline
\end{tabular}

The results of statistical analysis suggest there are distinctive signal patterns across different actions types, since all coefficients have statistically significant p-values (less than 0.05). One of the four EEG-vigilance assessment channel (F3) also shows significance in both models. In addition, the value of deviance of the fit indicate dummy variables of actions could greatly improve the model's accuracy.

\section{DISCUSSION AND CONCLUSIONS}

Measurement of construction workers' the ability of risk perception plays a vital role in safety management. This paper proposes to use EEG signals as the assessment tool to quantify the workers' vigilance and perceived risk level. The onsite experiment suggests the EEG signal from different spatial channels are sensitive to the variation in the EEGvigilance signals. Comparing to the current state-of-the-art, this proposed method is more objective and efficient for future development of automatic vigilance diagnose and hazard detection. The implementation of the proposed method can not only supplement the current observation approach but also suitable for post-hoc accident analysis as well.

\section{ACKNOWLEDGMENTS}

This work was financially supported by the National Natural Science Foundation of China (NSFC), \#51508487. Any opinions, findings, conclusions, or recommendations expressed in this paper are those of the authors and do not necessarily reflect the views of the NSFC.

\section{REFERENCES}

Arezes, P.M. \& Miguel, A.S., 2008. Risk perception and safety behaviour: A study in an occupational environment. Safety Science, 46(6), pp.900-907. 
Bohm, J. \& Harris, D., 2015. Risk Perception and Risk-Taking Behavior of Construction Site Dumper Drivers. International Journal of Occupational Safety and Ergonomics, 16(1), pp. 55-67.

Buzsaki, G., 2011. Rhythms of the Brain, Oxford University Press.

Chen, J., Song, X. \& Lin, Z., 2016. Revealing the "Invisible Gorilla" in construction: Estimating construction safety through mental workload assessment. Automation in Construction, 63, pp.173-183.

Cheng, E.W.L., Ryan, N. \& Kelly, S., 2012. Exploring the perceived influence of safety management practices on project performance in the construction industry. Safety Science, 50(2), pp.363-369.

Cohen, M., 2014. Analyzing neural time series data: theory and practice, $1^{\text {st }}$ Edition., Cambridge: The MIT Press.

Deery, H.A., 1999. Hazard and Risk Perception among Young Novice Drivers. Journal of Safety Research, 30(4), pp.225-236.

DeJoy, D.M., 2005. Behavior change versus culture change: Divergent approaches to managing workplace safety. Safety Science, 43(2), pp.105-129.

Demos, J., 2005. Getting started with neurofeedback, New York, NY, US: W. W. Norton \& Company.

Dzeng, R.-J., Lin, C.-T. \& Fang, Y.-C., 2016. Using eye-tracker to compare search patterns between experienced and novice workers for site hazard identification. Safety Science, 82, pp.56-67.

Fang, D., Zhao, C. \& Zhang, M., 2016. A Cognitive Model of Construction Workers' Unsafe Behaviors. Journal of Construction Engineering and Management, 142(9), p.4016039.

Hart, S. \& Staveland, L., 1988. Development of NASA-TLX (Task Load Index): Results of empirical and theoretical research. Advances in psychology, 52, pp.139-183.

Haslam, R.A. et al., 2005. Contributing factors in construction accidents. Applied Ergonomics, 36(4), pp.401-415.

Hegerl, U. et al., 2008. EEG-vigilance differences between patients with borderline personality disorder, patients with obsessive-compulsive disorder and healthy controls. European Archives of Psychiatry and Clinical Neuroscience, 258(3), pp.137-143.

Krallis, D. \& Csontos, A., 2006. From Risk Perception to Safe Behaviour, Available at: https://sia.org.au/downloads/SIGs/Resources/From_Risk_Perception_to_Safe_Behavi our.pdf.

Lin, C.-T. et al., 2012. Neurocognitive Characteristics of the Driver: A Review on Drowsiness, Distraction, Navigation, and Motion Sickness. Journal of Neuroscience and Neuroengineering, 1(1), pp.61-81.

Loomis, A.L., Harvey, E.N. \& Hobart, G.A., 1937. Cerebral states during sleep, as studied by human brain potentials. Journal of Experimental Psychology, 21(2), pp.127-144.

Mitropoulos, P. \& Namboodiri, M., 2010. New method for measuring the safety risk of construction activities: Task demand assessment. Journal of Construction Engineering and Management, 137(1), pp.30-38.

Niv, Y. et al., 2012. Neural Prediction Errors Reveal a Risk-Sensitive ReinforcementLearning Process in the Human Brain. Journal of Neuroscience, 32(2), pp.551-562.

Olbrich, S. et al., 2009. EEG-vigilance and BOLD effect during simultaneous EEG/fMRI measurement. NeuroImage, 45(2), pp.319-332.

Pfurtscheller, G. \& Silva, F. Da, 1999. Event-related EEG/MEG synchronization and desynchronization: basic principles. Clinical neurophysiology, 110(11), pp.1842-1857. 
Rethi, L.L. et al., 1999. Hazard recognition training program for construction, maintenance and repair activities,

Roth, B., 1961. The clinical and theoretical importance of EEG rhythms corresponding to states of lowered vigilance. Electroencephalography and Clinical Neurophysiology, 13(3), pp.395-399.

Sterman, M. \& Mann, C., 1994. Multiband topographic EEG analysis of a simulated visuomotor aviation task. International Journal of Psychophysiology, 16(1), pp.49-56.

Stewart, A.M. et al., 2012. Injured Workers' Construction of Expectations of Return to Work with Sub-Acute Back Pain: The Role of Perceived Uncertainty. Journal of Occupational Rehabilitation, 22(1), pp.1-14.

Suraji, A., Duff, A.R. \& Peckitt, S.J., 2001. Development of Causal Model of Construction Accident Causation. ASCE Journal of Consturction Engineering and Management, 127(4), http://dx.doi.org/10.1061/(ASCE)07339364(2001)127:4(337)\#sthash.fzr8swR9.dpuf .

Sweller, J., 1988. Cognitive load during problem solving: Effects on learning. Cognitive science, 12(2), pp.257-285.

Yang, K. et al., 2016. Semi-supervised near-miss fall detection for ironworkers with a wearable inertial measurement unit. Automation in Construction, 68, pp.194-202. 